\title{
Treatment of Chronic Fatigue Syndrome and Fibromyalgia with D-Ribose- An Open-label, Multicenter Study
}

\author{
Jacob Teitelbaum*, ${ }^{*}$, Janelle Jandrain ${ }^{2}$ and Ryan McGrew ${ }^{3}$
}

${ }^{1}$ Kona Research Center, 76-6326 Kaheiau St, Kailua Kona, Hawaii 96740, and Fibromyalgia and Fatigue Centers, Addison, Texas, USA; ${ }^{2,3}$ Schwabe North America, 3051 West Maple Loop Dr., Lehi, UT 84043, USA

\begin{abstract}
Objectives: Chronic Fatigue Syndrome and Fibromyalgia (CFS/FMS) are debilitating syndromes affecting 2$4 \%$ of the population. Although they are heterogeneous conditions associated with many triggers, they appear to have the common pathology of being associated with impaired energy metabolism.
\end{abstract}

As D-ribose has been shown to increase cellular energy synthesis, and was shown to significantly improve clinical outcomes in CFS/FMS in an earlier study, we hypothesized that giving D-ribose would improve function in CFS/FMS patients.

Design, Location, and Subjects: An open-label, unblinded study in which 53 US clinics enrolled 257 patients who had been given a diagnosis of CFS/FMS by a health practitioner.

Interventions: All subjects were given D-ribose $\left(\right.$ Corvalen $\left.^{\mathrm{TM}}\right)$, a naturally occurring pentose carbohydrate, 5-g TID for 3 weeks.

Outcome measures: All patients were assessed at baseline (1 week before treatment), and after 1,2, \& 3 weeks using a Visual Analog Scale(1-7 points) rating energy, sleep, cognitive function, pain and overall well being.

Results: 203 patients completed the 3 week treatment trial. D-ribose treatment led to both statistically $(\mathrm{p}<.0001)$ and clinically highly important average improvements in all categories:

- $61.3 \%$ increase in energy

- $37 \%$ increase in overall well being

- $29.3 \%$ improvement in sleep

- $30 \%$ improvement in mental clarity

- $15.6 \%$ decrease in pain

Improvement began in the first week of treatment, and continued to increase at the end of the 3 weeks of treatment. The D-ribose was well tolerated.

Conclusions: In this multicenter study, D-ribose resulted in markedly improved energy levels, sleep, mental clarity, pain relief, and well being in patients suffering from fibromyalgia and chronic fatigue syndrome. clinicaltrials.gov NCT01108549

Keywords: Fibromyalgia, chronic fatigue syndrome, energy levels, D-ribose, pain, pain relief.

\section{INTRODUCTION}

Fibromyalgia (FMS), which currently affects an estimated 3 to 6 million Americans [1, 2], and Chronic Fatigue Syndrome (CFS) are two overlapping and disabling syndromes. CFS affects more than one million people in the United States. There are tens of millions of people with similar fatiguing illnesses who do not fully meet the strict research definition of CFS [3]. Prevalence of these syndromes has been increasing rapidly over the last 2

*Address correspondence to this author at the 76-6326 Kaheiau St; Kailua Kona, Hawaii USA 96740; Fax: 808-329-5534;

E-mail : Endfatigue@aol.com decades, with European FMS prevalence rates in 5 countries being reported at $~ 3-5 \%$ [4], and CFS growing from .0002\% to as high as $2.5 \%$ in the US [5]. Severe persistent fatigue, diffuse migratory pain, cognitive dysfunction, and disordered sleep are common symptoms reported by patients suffering with these syndromes.

Many of the problems seen in CFS/FMS may be associated with a decrease in tissue energy levels and altered energy metabolism. The consequences of dysfunctional energy metabolism frequently include pain from chronic muscle shortening [6], postexertional fatigue, and low exercise tolerance associated with decreased blood cell mass, cardiac output and stroke volumes [7, 8]. Adenosine 
triphosphate (ATP) levels have also been shown to be $80 \%$ higher in healthy vs. CFS patients [9]. In addition, it has been suggested that decreased energy production also results in hypothalamic dysfunction, which can result in the disordered sleep, hormonal imbalances, and autonomic dysfunctions seen in these syndromes [10]. Many causes and mechanisms for mitochondrial dysfunction have been proposed, including an alteration in muscle adenine nucleotide metabolism affecting ATP levels and depleting energy reserves [11,12].

D-ribose, a naturally occurring pentose carbohydrate, is a key structural component of the DNA, RNA, ATP, FADH, Coenzyme-A, and NADH needed by the mitochondria to maintain cellular energy stasis. D-ribose was shown in earlier studies to be very helpful in improving clinical outcomes in those with CFS and FMS [13] and in a general population of people with fatigue [14], and has also been reported to be effective in restoring tissue energy levels following exercise [15]. Because of these known benefits, it was hypothesized that D-ribose would increase energy production, and therefore decrease symptoms, in patients suffering from FMS and CFS. As the initial study [13] was done by a single practitioner, and CFS and FMS appear to be heterogeneous syndromes, this study was designed to test whether the benefits seen in the earlier study could be generalized to the CFS/FMS population at large, by doing a multicenter study recruiting and treating patients at 53 health care practitioners' offices throughout the United States.

\section{MATERIALS AND METHODS}

\section{Patient Enrollment}

Fifty-three healthcare practitioners, practicing throughout the US, enrolled 257 patients who had been diagnosed with Fibromyalgia (FMS) and/or Chronic Fatigue Syndrome. All practitioners who purchased the Corvalen from Integrative Therapeutics, Inc. were invited to participate and invite patients who satisfied the entrance criteria below to volunteer for the study. Being a pilot study, where we were looking for widespread inclusion representing a broad patient and practitioner base, with neither of these receiving any payment for their involvement, the design (an open, unblinded trial) and outcome measure instruments were kept very simple to improve compliance.

Volunteers received information about the study product, Corvalen ${ }^{\mathrm{TM}}$ D-ribose, its potential benefits, and possible side-effects. All patients gave informed consent, and the study was approved by the ITI research review committee. The patients received free product for the study, but were otherwise not financially compensated for their participation.

Inclusion criteria:

1) Over 18 yo. Not limited by gender or race.

2) Diagnosed with Fibromyalgia (FMS) (defined by American College of Rheumatology [ACR] criteria) and/or Chronic Fatigue Syndrome (CFS- by Centers for Disease Control [CDC] criteria) by a health practitioner.

Exclusion Criteria:
1) Having used D-ribose at over 5 grams a day for at least 3 weeks in the past or any D-ribose containing products in the 7 days prior to the study.

2) The study did not include any pregnant or nursing women or any participants with known severe medication or nutrient sensitivities. Otherwise, as we chose to have a cohort representative of the population carrying a diagnosis of CFS and/or FMS as a whole, including those with secondary fibromyalgia, patients with other medical conditions were included. Therefore, evaluation to rule out other conditions was not done, nor were subjects excluded who were on other treatments.

\section{Formula and Dosage}

The practitioners directed patients to use D-ribose $\left(\right.$ Corvalen ${ }^{\mathrm{TM}}$ ) at a dose of 5 grams, three times a day, for 3 weeks. They were instructed that the powder was to be mixed with food, water, or another beverage.

\section{Experimental Design}

This was an open, unblinded trial. After confirming that the subjects met the entry criteria, the practitioners completed an optional information sheet including a demographic summary, whether the patient had CFS, FMS or both, and recorded supplements, over-the-counter drugs (OTCs) and prescription drugs that the patient used regularly. The practitioners gave subjects the study product, a baseline questionnaire, three weekly follow up questionnaires, and four prepaid and addressed envelopes. Patients were allowed to take their other treatments in addition to the D-ribose.

\section{Outcome Measures}

The patients rated five FMS/CFS symptoms at baseline ( 1 week before they began treatment), and after 1, 2, and 3 weeks of treatment using a 7-point hedonic scale. The assessed symptoms were energy level, sleep quality, mental clarity, pain level, and overall sense of well being (based on the subjects' individual interpretation of these symptoms and using the guidelines given to them- see symptom scales in Table 1). Average scores with $1 \mathrm{SD}$ were calculated for each of the 5 symptoms at baseline and weeks 1,2 , and 3 . P values were measured relative to the baseline period.

The patients answered (on the questionnaire) if they had taken the directed dosage after each week of treatment and if they experienced any adverse side effects.

\section{RESULTS}

\section{Compliance}

Thirty-one of the 257 patients who entered the study and completed 1 week of use did not complete 2 weeks of use, and another 23 patients did not complete 3 weeks of use, with 203 patients completing the study. Four of the 257 patients noted that they discontinued the D-ribose because of adverse effects (see the adverse effects section). No other patients reported reasons for discontinuing the study. All patients who completed week 2 of the treatment study questionnaires were included in the study analysis. 201 patients completed self-evaluation for compliance with the dosing instructions. Of those, 117 (58\%) patients reported 
Table 1.D-Ribose Questionnaire, 7-Point Hedonic Scale Questions

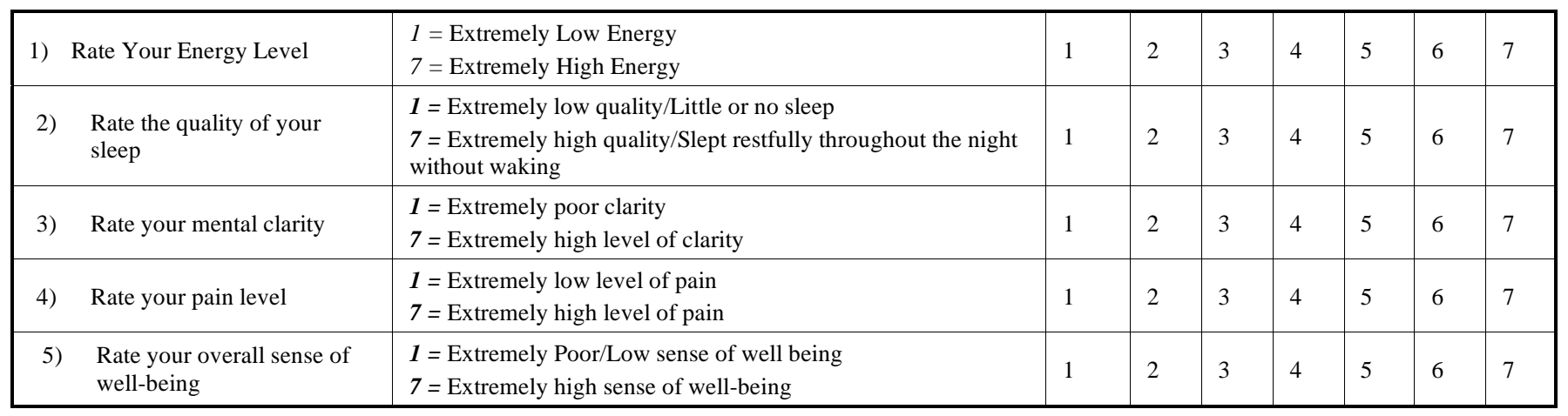

Table 2. Patient Demographics

\begin{tabular}{|l|l|l|}
\hline Patients assessed for CFS/FMS (n=163) & & \\
\hline \hline Patients with CFS & 53 & $32.5 \%$ \\
\hline Patients with FMS & 67 & $41.1 \%$ \\
\hline Patients with CFS and FMS & 43 & $26.4 \%$ \\
\hline Patients assessed for age(n=162) & & \\
\hline Average Age (years) & 51 & - \\
\hline Age (Range) & $25-90$ & \\
\hline Patients assessed for gender(n=166) & & 14 \\
\hline Male & 152 & $8.4 \%$ \\
\hline Female & 152 & $91.6 \%$ \\
\hline
\end{tabular}

full compliance, and 171 (85\%) patients reported using at least 2 doses of 5 grams every day throughout all 3 weeks of product use.

\section{Study Population}

Demographic information was collected from a representative sample, which included over $60 \%$ of the enrolled study population (see Table 2).

\section{Primary Outcome Measures}

Significant improvements in all 5 primary outcome measures (energy, sleep, cognitive function, pain and overall well being) were seen after 1 week of product use $(\mathrm{p}<0.0001$ for all). The improvement increased through the second and third week of product use ( $<<0.0001$ for all). After 3 weeks of product use, the average improvements in energy levels was $61 \%$, overall well being $37 \%$, sleep $29 \%$, mental clarity $30 \%$, and pain decreased an average of $16 \%$. See Table 3 and Fig. (1) below. Outcome measures broken down by groups "CFS only", "FMS only" and "both CFS \& FMS" showed similar outcomes to the group as a whole except for the "FMS group" showing significant differences in pain relief compared to the total cohort (see Table 3) .

\section{Adverse Effects}

Two of the patients who enrolled in the study cited that they discontinued use because they became ill (unrelated to product use). Another patient recorded that they discontinued use during the second week of product use because of nausea. One more patient indicated that they discontinued use because of increased insomnia.

\section{DISCUSSION}

This study supports earlier research in subjects with CFS and/or Fibromyalgia [13] showing that using D-ribose significantly increased energy, sleep quality, mental clarity, and well being, while also decreasing pain. The earlier study was done in subjects recruited by a single physician, and experience has suggested that CFS and FMS are very heterogeneous conditions. This study was done to assess whether the earlier pilot study findings would be confirmed, and also could be generalized to a broader population, by performing a multicenter trial including 53 health practitioners. It is very promising that this safe and relatively low cost treatment was able to significantly improve patients' clinical outcomes, with subjects reporting an average $61 \%$ increase in energy after only 3 weeks, and significant improvement beginning at the 1 week follow up.

Why might D-ribose be beneficial in CFS/FMS? Patients with FMS [16, 17] and /or CFS [18] generally demonstrate reduced sustained and next day exercise capacity, requiring much longer to recover from exercise (postexertional fatigue). These problems are frequently associated with abnormal metabolism, and many FMS studies have been designed to investigate alterations in muscle metabolism using both traditional biopsy techniques [11] and nuclear magnetic resonance spectroscopy (P-31 MRS) [19-24]. Decreased ATP concentrations with accompanying changes in energy metabolism have also been found in the red blood 
Table 3. Baseline and Post-Weeks 1, 2, and 3 Assessments for All Patients, CFS Patients, FMS Patients, and Patients with Both CFS and FMS

\begin{tabular}{|c|c|c|c|c|c|}
\hline All Patients & Baseline & Week $2(\mathrm{~N}=257)$ & Week $3(N=226)$ & Week $4(\mathrm{~N}=203)$ & $\begin{array}{l}\text { Improvement After } 3 \\
\text { Weeks of Use }\end{array}$ \\
\hline Energy & 2.53 & 3.37 & 3.80 & 4.08 & 1.55 \\
\hline Sleep & 3.21 & 3.70 & 3.91 & 4.15 & 0.94 \\
\hline std & 1.36 & 1.36 & 1.31 & 1.38 & $29 \%$ \\
\hline Mental clarity & 3.38 & 3.85 & 4.19 & 4.39 & 1.01 \\
\hline Pain & 4.63 & 4.18 & 3.96 & 3.91 & -0.73 \\
\hline std & 1.54 & 1.47 & 1.47 & 1.49 & $-16 \%$ \\
\hline Well-being & 3.05 & 3.67 & 3.98 & 4.18 & 1.13 \\
\hline std & 1.16 & 1.20 & 1.25 & 1.32 & $37 \%$ \\
\hline \multicolumn{6}{|l|}{ CFS patients } \\
\hline & Baseline & Week $2(\mathrm{~N}=53)$ & Week $3(\mathrm{~N}=47)$ & Week $4(\mathrm{~N}=43)$ & $\begin{array}{l}\text { Improvement After } 3 \\
\text { Weeks of Use }\end{array}$ \\
\hline Sleep & 3.64 & 4.04 & 4.02 & 4.30 & 0.66 \\
\hline std & 1.30 & 1.34 & 1.41 & 1.28 & $18 \%$ \\
\hline Mental clarity & 3.55 & 3.81 & 4.17 & 4.33 & 0.78 \\
\hline std & 1.14 & 1.23 & 1.32 & 1.25 & $22 \%$ \\
\hline Pain & 3.92 & 3.77 & 3.53 & 3.40 & -0.53 \\
\hline std & 1.25 & 0.00 & 1.37 & 1.50 & $-13 \%$ \\
\hline Well-being & 3.28 & 3.66 & 3.81 & 4.28 & 1.00 \\
\hline std & 1.26 & 1.24 & 1.30 & 1.18 & $30 \%$ \\
\hline \multicolumn{6}{|l|}{ FMS Patients } \\
\hline & Baseline & Week $2(\mathrm{~N}=67)$ & Week $3(\mathrm{~N}=62)$ & Week $4(\mathrm{~N}=55)$ & $\begin{array}{l}\text { Improvement After } 3 \\
\text { Weeks of Use }\end{array}$ \\
\hline std & 1.27 & 1.33 & 1.38 & 1.40 & $-15 \%$ \\
\hline Well-being & 3.16 & 3.75 & 4.16 & 4.29 & 1.13 \\
\hline std & 1.05 & 1.17 & 1.15 & 1.24 & $36 \%$ \\
\hline \multicolumn{6}{|c|}{ Patients with FMS and CFS } \\
\hline & Baseline & Week $2(\mathrm{~N}=43)$ & Week $3(\mathrm{~N}=38)$ & Week $4(\mathrm{~N}=32)$ & $\begin{array}{l}\text { Improvement After } 3 \\
\text { Weeks of Use }\end{array}$ \\
\hline Energy & 2.28 & 3.23 & 3.58 & 4.25 & 1.97 \\
\hline std & 0.96 & 1.32 & 1.39 & 1.63 & $86 \%$ \\
\hline Sleep & 2.88 & 3.33 & 3.68 & 4.22 & 1.34 \\
\hline std & 1.38 & 1.48 & 1.51 & 1.45 & $46 \%$ \\
\hline Mental clarity & 3.40 & 3.70 & 4.21 & 4.69 & 1.29 \\
\hline std & 1.43 & 1.34 & 1.42 & 1.53 & $38 \%$ \\
\hline Pain & 4.88 & 4.37 & 4.26 & 4.13 & -0.76 \\
\hline std & 1.71 & 1.57 & 1.73 & 1.52 & $-16 \%$ \\
\hline Well-being & 2.74 & 3.51 & 3.97 & 4.19 & 1.44 \\
\hline std & 1.16 & 1.35 & 1.57 & 1.64 & $53 \%$ \\
\hline
\end{tabular}

cells and neutrophils of CFS [25] and fibromyalgia [26] patients, suggesting that the energy deficits seen in in these syndromes may be widespread.
Adenine nucleotide adenosine triphosphate (ATP) is the primary energy source of all living cells. In tissues subjected to the metabolic stress of hypoxia, ischemia, or 


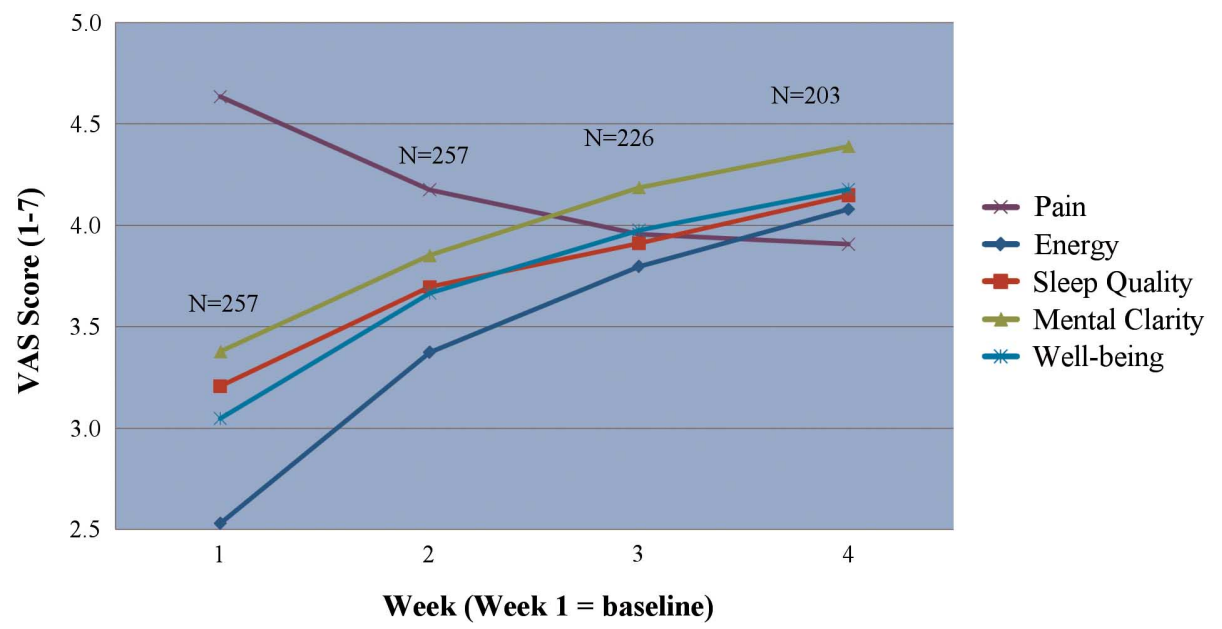

Fig. (1). Average hedonic rating of 5 symptoms from baseline through 3 weeks of daily D-ribose use.

mitochondrial dysfunction, ATP is broken down and the ability to recycle expended energy is disrupted. As such, adenosine diphosphate (ADP) levels accumulate leading to a series of reactions in the cell to balance ATP/ADP ratios and maintain energy stasis. These reactions ultimately lead to increased intracellular concentrations of adenosine monophosphate (AMP). In an effort to control energy balance, cells catabolize AMP in reactions catalyzed by 5'nucleotidase (heart) and AMP deaminase (skeletal muscle) ultimately forming inosine, hypoxanthine and adenine. These catabolic end products are washed out of the cell netting a loss of purines and a reduction in the total pool of adenine nucleotides available to the tissue, lowering its phosphorylation potential. Up to $90 \%$ of these catabolites can potentially be biochemically salvaged and recycled [15, $27,28]$. Supplemental D-ribose bypasses the rate limiting enzymes of the pentose phosphate pathway ( PPP), going directly to purine synthesis. D-ribose has been shown to accelerate energy recovery in skeletal muscle and to relieve fatigue, soreness, and stiffness after intense exercise [15, 27, 28]. This could explain D-ribose's mechanism of action in CFS and Fibromyalgia, and the biochemistry of D-ribose is discussed in more detail in our earlier study [13].

D-ribose has been extensively studied in both heart and muscle. Safety data is well accepted, with no significant adverse reactions. Investigators have concluded that D-ribose is well tolerated at dosages of up to $60 \mathrm{~g} /$ day $[29,30]$.

This study has several limitations, one of which is the lack of a placebo control group. Doing an RCT will be the next key step, but in the interim it was considered important to see whether the clinical benefits seen in the earlier study would also occur in a larger and multicenter study.

A second limitation is that the inclusion criteria simply required that the subject had been diagnosed with CFS or FMS by a healthcare practitioner, and did not require repeating the testing needed to rule out other conditions. It also did not proscribe the use of other treatments. This study, however, was meant to be a clinical study testing simply whether people in the general public who carried a diagnosis of CFS or FMS would improve with D-ribose, while receiving their concurrent care. Also, unlike CFS, underlying other diagnoses do not exclude a diagnosis of fibromyalgia.
A third limitation is that only subjective outcome measures were used. The diagnoses of FMS and CFS are largely subjective, however, as there are no laboratory tests that have adequate sensitivity and specificity to be generally accepted as disease marker(s). As a result, diagnosis (as well as the patient's disability) is based on subjective criteria and these criteria were also therefore used as our outcome measures.

The fourth concern is that 3 weeks may not be long enough to achieve steady-state, maximum treatment effects/benefits from the treatment. In future research, the researchers should consider evaluating patients for a longer period, to see when benefits level off, and to confirm that they persist.

\section{CONCLUSIONS}

This follow up study reproduced the benefits of D-ribose seen in our earlier study. As it was a multicenter study involving 53 health practitioners and a larger number of patients, it also suggests that these findings will apply to many of the diverse subsets seen in this patient population.

That subjects reported an average $61 \%$ increase in energy and $37 \%$ improvement in overall well being, along with improved cognitive function and sleep (and decreased pain), is encouraging. Although it is important to do a randomized, placebo controlled trial with a longer treatment period, these 2 preliminary studies suggest that D-ribose may be a promising therapeutic option for patients with CFS and FMS, offering a significant improvement in their quality of life.

\section{ABBREVIATIONS}

ADP

$=$ adenosine diphosphate

AMP

ATP

CFS

$=$ adenosine monophosphate

DNA

FADH

$=$ Adenosine triphosphate

$=$ Chronic fatigue syndrome

FMS

NADH
$=$ Deoxyribonucleic acid

$=$ Flavin adenine dinucleotide

$=$ Fibromyalgia

$=$ Nicotinamide adenine dinucleotide 
PPP = Pentose phosphate pathway

RNA $\quad=$ ribonucleic acid

\section{CONFLICT OF INTEREST}

None declared

\section{ACKNOWLEDGEMENTS}

We would like to thank the practitioners and others that made this study possible.

\section{AUTHOR DISCLOSURE STATEMENT}

Teitelbaum notes that he sells natural supplements, including ribose, on his website (ribose is widely available). All of his royalties for products he designs or work with go to charity, including the Teitelbaum Family Foundation. Jandrain and McGrew are employees of Schwabe North America, which funded this study. Practitioners involved in the study received no payment for being part of the study, and were customers of (rather than employees of) Integrative Therapeutics.

\section{REFERENCES}

[1] Goldenberg DL. Fibromyalgia syndrome-an emerging but controversial condition. JAMA 1987; 257: 2782-7.

[2] Wolfe F, Ross K, Anderson J, Russell IJ, Hebert L. The prevalence $\&$ characteristics of fibromyalgia in the general population. Arthritus Rheum 1995; 38(1): 19-28.

[3] Leven MV, Zielhuis GA, Jos W, Meer JW, Verbeek AL, Bleijenberg G. Fatigue and chronic fatigue syndrome-like complaints in the general population. Eur J Public Health 2010; 20: 251-7.

[4] Branco JC, Bannwarth B, Failde I, et al. Prevalence of fibromyalgia: a survey in five European countries. Semin Arthritis Rheum 2010; 39: 448-53.

[5] Jason L. A constructive debate with the CDC on the empirical case definition of chronic fatigue syndrome. J Disabil Policy Stud 2010; 20: 251.

[6] Travell JG, Simons DG. Myofascial pain and dysfunction: the trigger point manual. Baltimore, MD: Williams \& Wilkins 1983, Vol. I, pp. 103-64.

[7] Peckerman A. Abnormal impedance cardiography predicts symptom severity in chronic fatigue syndrome. Am J Med Sci 2003; 26(2): 55-60.

[8] Hurwitz BH, Coryell VT, Parker M, et al. Chronic fatigue syndrome: illness severity, sedentary lifestyle, blood volume and evidence of diminished cardiac function. Clin Sci (Lond) 2009; 118(2): 125-35.

[9] Bazzichi L, Giannaccini G, Betti L. ATP, calcium and magnesium levels in platelets of patients with primary fibromyalgia. Clin Biochem 2008; 41(13): 1084-90.

[10] Teitelbaum JE, Bird B, Greenfield RM. Effective treatment of CFS and fms: a randomized, double-blind placebo controlled study. J Chronic Fatigue Syndr 2001; 8(2): 3-24.

[11] Bengtson A, Heriksson KG, Larsson J. Reduced high-energy phosphate levels in the painful muscles of patients with primary fibromyalgia. Arthritis Rheum 1986; 29(7): 817-21.
[12] Eisinger J, Plantamura A, Ayavou T. Glycolysis abnormalities in fibromyalgia. J Am Coll Nutr 1994; 13(2): 144-8.

[13] Teitelbaum JE, St Cyr JA, Johnson C. The use of D-ribose in chronic fatigue syndrome and fibromyalgia: a pilot study. J Altern Complement Med 2006; 12(9): 857-62.

[14] MacCarter D, Flanigan R, Washam M, Shecterle L, St Cyr J. D-Ribose Improves Fatigue in Adults. Presented at the Scripps Integrative Conference Poster Presentation, January 24, 2009.

[15] Hellsten Y, Skadhauge L, Bangsbo J. Effect of -ribose supplementation on resynthesis of adenine nucleotides after intense intermittent training in humans. Am J Physiol Regul Integr Comp Physiol 2004; 286(1): R182-8.

[16] Lund N, Bengtsson A, Thorborg P. Muscle tissue oxygen in primary fibromyalgia. Scan J Rheumatol 1986; 15(2): 165-73.

[17] Schachter CL, Busch AL, Peloso PM, Shepard MS. Effects of short versus long bouts of aerobic exercise in sedentary women with fibromyalgia: a randomized controlled trial. Phys Ther 2003; 83(4): 340-58.

[18] Vanness JM, Stevens SR, Bateman L, Stiles TL, Snell CR. Postexertional malaise in women with chronic fatigue syndrome. J Womens Health (Larchmt) 2010; 19(2): 239-44.

[19] Lund E, Kendall SA, Janerot-Sjoberg B, Bengtsson A. Muscle metabolism in fibromyalgia studied by P-31 magnetic resonance spectroscopy during aerobic and anaerobic exercise. Scan J Rheumatol 2003; 32(3): 138-45.

[20] Strobl ES, Krapf M, Suckfull M, Bruckle W, Fleckenstein W, Muller W. Tissue oxygen measurement and 31P magnetic resonance spectroscopy in patients with muscle tension and fibromyalgia. Rheumatol Int 1997; 16(5): 175-80.

[21] Sprott H, Rzanny R, Reichenbach JR, Kaiser WA, Hein G, Stein G. $31 \mathrm{P}$ magnetic resonance spectroscopy in fibromyalgic muscle. Rheumatol (Oxford) 2000; 39(10): 1121-5.

[22] Park JH, Phothimat P, Oates CT, Hernanz-Schulman M, Olson NJ. Use of P-31 magnetic resonance spectroscopy to detect metabolic abnormalities in muscles of patients with fibromyalgia. Arthritis Rheum 1998; 41(3): 406-13.

[23] Jacobsen S, Jensen KE, Thomsen C, Danneskiold SB, Henriksen O. Magnetic resonance spectroscopy in fibromyalgia. A study of phosphate-31 spectra from skeletal muscles during rest and after exercise. Ugeskr Laeger 1994; 156(46): 6841-4.

[24] Kushmerick MJ. Muscle energy metabolism, nuclear magnetic resonance spectroscopy and their potential in the study of fibromyalgia. J Rheumatol 1989; 19: 40-6.

[25] Myhill S, Booth NE, McLaren HJ. Chronic fatigue syndrome and mitochondrial dysfunction. Int J Clin Exp Med 2009; 2: 1-16.

[26] Eisinger J, Bagneres D, Arroyo P, Plantamura A, Ayavou T. Effects of magnesium, high-energy phosphates, piracetam and thiamin on erythrocyte transketolase. Magnes Res 1994; 7(1): 5961 .

[27] Brault JJ, Terjung RL. Purine salvage to adenine nucleotides in different skeletal muscle fiber types. J Appl Physiol 2001; 91: 2318.

[28] Tullson PC, Terjung RL. Adenine nucleotide synthesis in exercising and endurance-trained skeletal muscle. Am J Physiol 1991; 261: C342-7.

[29] Gross M, Dormann B, Zollner N. D-ribose administration during exercise: effects on substrates and products of energy metabolism in healthy subjects and a patient with myoadenylate deaminase deficiency. Klin Wochenschr 1991; 69: 151-5.

[30] Pliml W, von Arnim T, Stablein A, Hofmann H, Zimmer HG, Erdmann E. Effects of D-ribose on exercise-induced ischaemia in stable coronary artery disease. Lancet 1992; 340(8818): 507-10. 